\title{
Agromorphological Characterization and Nutritional Value of Traditional Almond Cultivars Grown in the Central-Western Iberian Peninsula
}

\author{
Rodrigo Pérez-Sánchez *(D) and María Remedios Morales-Corts (D) \\ Faculty of Agricultural and Environmental Sciences, University of Salamanca, Avda. Filiberto Villalobos, 119, \\ 37007 Salamanca, Spain; reme@usal.es \\ * Correspondence: rodrigopere@usal.es
}

Citation: Pérez-Sánchez, R.; Morales-Corts, M.R.

Agromorphological Characterization and Nutritional Value of Traditional Almond Cultivars Grown in the Central-Western Iberian Peninsula. Agronomy 2021, 11, 1238. https:// doi.org/10.3390/agronomy11061238

Academic Editors:

Gregorio Barba-Espín, José

Ramón Acosta-Motos and Essaid Ait Barka

Received: 5 May 2021

Accepted: 16 June 2021

Published: 18 June 2021

Publisher's Note: MDPI stays neutral with regard to jurisdictional claims in published maps and institutional affiliations.

Copyright: (c) 2021 by the authors. Licensee MDPI, Basel, Switzerland. This article is an open access article distributed under the terms and conditions of the Creative Commons Attribution (CC BY) license (https:// creativecommons.org/licenses/by/ $4.0 /)$.

\begin{abstract}
In this study, 24 traditional almond cultivars grown in the central-western Iberian Peninsula, all of them clearly in decline or close to extinction, were characterized from the agromorphological and chemical points of view. A total of 40 agromorphological and chemical descriptors, mainly defined by the IPGRI and the UPOV, were used to describe the flowers, leaves, fruits and the trees themselves over three consecutive years (2015-2017). Some of the cultivars showed distinctive and interesting agronomical characteristics from a commercial point of view, such as high yields and high quality fruit. This was the case of the almond cultivars called "Gorda José" and "Marcelina". Their fruits were quite heavy (nuts: $>9.1 \mathrm{~g}$; kernels: $>1.9 \mathrm{~g}$ ), with very low percentages of double kernels $(<3 \%)$ and high nutritional value ( $>50 \%$ lipids; $>21 \%$ proteins). The results of the PCA and cluster analysis showed that agromorphological and chemical analysis can provide reliable information on the variability in almond genotypes. This work constitutes an important step in the conservation of genetic almond resources in the central-western Iberian Peninsula.
\end{abstract}

Keywords: almond descriptors; conservation; endangered cultivars; fruit quality; genetic resources; Prunus dulcis

\section{Introduction}

The almond (Prunus dulcis [Miller] D.A. Webb syn. P. amygdalus (L.) Batsch, Rosaceae, $2 n=2 x=16$ ) is one of the oldest and most important nut crops grown commercially worldwide. It originated in the arid mountainous regions of southwestern and central Asia [1] and spread rapidly towards the Mediterranean Basin via seeds carried by caravans along the old Silk Route [2]. The almond is cultivated for its edible seed (the kernel), which is used for direct consumption and for almond-based products and confections [3,4]. In 2019, world production of almonds was 3.49 million metric tons [5]. The United States, Spain, Iran, Australia, Morocco and Syria are the most important almond-producing countries (approximately 80\% of world almond production). Concretely, the Iberian Peninsula (the first European producer) has 717,870 ha dedicated to almond production and produces 373,970 metric tons of fruit per year. The main almond-producing regions in the Iberian Peninsula are close to the Mediterranean Sea, such as Andalusia, Murcia and Valencia, but also include inland regions such as Aragón and Castilla-La Mancha. In these regions, most of the almond orchards are not irrigated (92.2\%), resulting in very low productivity [6]. Some of the most common cultivars cultured in the Iberian Peninsula are "Marcona", "Desmayo Largueta", "Tuono", "Cristomorto", "Ferragnés", "Ferraduel",

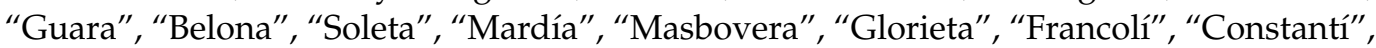
"Marinada", "Tarraco", "Vayro", "Parada", "Bonita" and "Casanova". Moreover, many other named cultivars of local origin have evolved from localized ecological niches that are found in different valleys extending inland from the Mediterranean coast [7-9]. The conservation and characterization of these local cultivars is important to avoid the loss of 
genetic variability and as a potential source of genetic variation for future almond breeding programs. Some of these cultivars show distinctive agronomic characteristics, such as self-compatibility, late blooming, frost tolerance and shell hardness.

Many studies addressing the agromorphological and biochemical characterization of almond cultivars have been undertaken in countries mainly located around the Mediterranean basin [10-24]. In the Iberian Peninsula, studies have been carried out by MonteroRiquelme et al. [25], Felipe [26], Cordeiro et al. [27], Arquero et al. [28], Kodad and Socias i Company [29,30], Vargas et al. [31], Kodad et al. [32-34] and Ramos and Costa [35].

The objective of the present study was to survey, identify and characterize from the agromorphological and nutritional points of view the traditional almond cultivars existing in the central-western region of the Iberian Peninsula in order to avoid their disappearance and so that they can be included in future almond breeding programs.

\section{Materials and Methods}

\subsection{Plant Material}

A survey was carried out in the regions known as "Arribes del Duero" (Salamanca, Spain) and "Trás-os-Montes e Alto Douro" (Bragança, Portugal) during 2014. A total of 192 adult almond trees at least ten years old, corresponding to 24 cultivars ("Agapitina", "Bravía”, "Cascafina”, “Cascona", "Cornicabra”, "De Convite”, “Desmayo Largueta”, "Desmayo Rojo", "Esperanza”, “Gorda José", "Marcelina”, "Marcona”, "Molar de Chavés”, "Mollar de Arribes", "Pestaneta de Bragança", "Pestañeta de Arribes", "Peta", "Picuda”, "Portuguesa", "Recia", "Redondilla", "Valenciana", "Verdeal" and "Verdinal"), were selected for study at full fruit maturity. Eight trees were evaluated per cultivar.

\subsection{Agromorphological Characterization}

The agromorphological description of the almond cultivars was based on 19 descriptors established by the UPOV [36] and IPGRI [37] and a further 14 descriptors that were considered relevant for identification. For the determination of some of the descriptors, samples of flowers, fruits and leaves were taken during 2015, 2016 and 2017, using UPOV guidelines. The measurement for each parameter was performed as follows: Flowers were collected at full bloom. Ten flowers were taken from each of the eight trees studied per cultivar and year, and the following quantitative parameters were measured using an electronic digital caliper (COMECTA 5900603, Barcelona, Spain): open flower diameter (cm), petal length $(\mathrm{cm})$, petal width $(\mathrm{cm})$ and pistil length $(\mathrm{cm})$. The numbers of petals, pistils and stamens were also counted. Leaves were collected at the adult stage, at approximately the end of July. Seven leaves were sampled per tree and year, and the following quantitative parameters were measured: petiole length and leaf blade length and width. Anthocyanin pigmentation of the nectaries was indicated by a reddish tonality observed in leaf glands. Two ratios were calculated: the length/width of the leaf blade and the petiole length/leaf blade length. Almond fruits were collected at maturity. The time of maturity was reached when the mesocarp started to dry off. All observations on dry fruits and kernels were made when the ripe fruits had a water content of less than $8 \%$; that is, at least one month after harvesting. A total of 20 fruits per tree and year were taken to determinate each parameter. The three principal dimensions of the nut and kernel, namely length (L), width (W) and thickness $(\mathrm{T})$, were measured using a digital caliper with a sensibility of $0.01 \mathrm{~mm}$. The geometric mean diameter $(\mathrm{Dg})$, sphericity $(\varnothing)$ and surface area $(\mathrm{S})$ were calculated using the following equations: $\mathrm{Dg}=(\mathrm{LWT})^{0.333}, \varnothing=\left[(\mathrm{LWT})^{0.333}\right] / \mathrm{L}$ and $\mathrm{S}=\pi \mathrm{Dg}^{2}$ [38-41]. Also, following Jain and Bal [42] and Özgüven and Vursavuş [43], the volume (V) was expressed as follows: $V=\pi B^{2} L^{2} / 6(2 L-B)$, where $B=(W T)^{0.5}$. Shell hardness was evaluated according to the categories established in the guidelines (extremely hard, hard, intermediate, soft and paper). Mass was measured on an electronic balance (Mettler XPR603S, Toledo, Spain) with a sensitivity of $\pm 0.001 \mathrm{~g}$. The percentage of doubles (number of kernels per nut) and kernel taste were also determined. Finally, with regard to whole trees, the vegetative bearing habit 
of the different cultivars was evaluated by considering growing habits from extremely upright to drooping.

\subsection{Chemical Composition}

One hundred almonds were randomly selected per cultivar and their shells were removed to obtain the kernel. They were then finely ground by an electric grinder (Moulinex MC3001, Barcelona, Spain) and analyzed, with three replicates, for the following parameters: dry weight, lipids, proteins, dietary fiber, carbohydrates and ash (AOAC procedures [44]). Dry matter (\%) was determined in a drying oven (Indelab, mod. IDL.AI 80, Navarra, Spain) at $100{ }^{\circ} \mathrm{C}$ using 25 g per sample. The crude protein content (\%) of the samples was estimated with the macro-Kjeldahl method, with sulfuric acid digestion (BlocDigest 12P SELECTA, Barcelona, Spain) performed prior to the distillation and titration process, which was carried out with IDK132 VELP equipment and a conversion factor (NProtein) of 5.18. The crude fat/lipids content (\%) was determined with a Soxhlet extractor (VELP SER-158 Technilab, Lisses, France) by extraction from a $5 \mathrm{~g}$ sample of powdered almond with petroleum ether (boiling point range $=38.2-54 \cdot 3^{\circ} \mathrm{C}$ ). The ash content $(\%)$ was determined by quantifying the residue after combustion of the dry sample in an analogical muffle furnace (HD230PAD Hobersal, Barcelona, Spain) at $550 \pm 15^{\circ} \mathrm{C}$ for 6 hours under incineration conditions corresponding to the gravimetric method. Total dietary fiber (\%) was measured with an enzymatic-gravimetric method, determining the fiber's hydrolyzed polysaccharides by HPLC and lignin gravimetrically. Total carbohydrates (\%) were estimated through the difference between the dry extract and the rest of the components. The energy value $(\mathrm{kcal} / 100 \mathrm{~g})$ was calculated using the general Atwater coefficients: $4 *(\%$ protein $)+9 *(\%$ crude fat $)+4 *$ (\%total carbohydrates $)$.

\subsection{Statistical Analyses}

Means and standard deviations were calculated for each of the quantitative parameters studied over the 3 years for the 24 traditional almond cultivars. The unit of measurement of each of the parameters studied was based on the individual value of each of the eight trees sampled per cultivar. Finally, based on all the studied parameters, principal component analysis (PCA) was carried out using the SPSS 17.0 program, and a dendrogram of genetic similarities among cultivars was compiled using the furthest neighbor method (Statgraphics Plus 17.0 program).

\section{Results}

\subsection{Flowers}

Flower parameters are shown in Table 1. Open flower diameter ranged from 3.48 to $5.51 \mathrm{~cm}$, the cultivars with the largest flowers being "Peta", "Marcelina", "Gorda José" and "Portuguesa". All genotypes had five petals, except "Picuda" and the "Pestañetas" group.

In all cases, the petal length was highly correlated (0.91) with the flower size. Petal width varied from 1.11 to $2.04 \mathrm{~cm}$. The lowest values were observed for "Pestañeta Arribes", "Desmayo Rojo" and "Esperanza". These last two cultivars, together with "Cascafina", "Cascona", "De Convite", "Desmayo Largueta" and "Peta", showed more than one pistil. The length of pistils ranged from 1.26 to $2.05 \mathrm{~cm}$. "Portuguesa" was the cultivar with the largest pistil. The stamen number varied between 28 and 53 . 
Table 1. Means, standard deviations and ANOVA analyses for some flower parameters in almond cultivars.

\begin{tabular}{|c|c|c|c|c|c|c|c|}
\hline Cultivar & $\begin{array}{c}\text { Open Flower } \\
\text { Diameter }(\mathrm{cm})\end{array}$ & Number of Petals & Petal Length (cm) & Petal Width (cm) & Number of Pistils & Pistil Length $(\mathrm{cm})$ & Number of Stamens \\
\hline Agapitina & $4.38(0.18)$ defg & 5 & $1.89(0.04)^{c}$ & $1.27(0.03)^{\mathrm{cd}}$ & 1 & $1.83(0.03) \mathrm{hi}$ & $32.28(1.97)$ bcdef \\
\hline Bravía & $4.35(0.14)^{\text {cdef }}$ & 5 & $1.72(0.07)^{b}$ & $1.61(0.04)^{h}$ & 1 & $1.26(0.05)^{\mathrm{a}}$ & $31.06(3.01)$ abcdef \\
\hline Cascafina & $4.91(0.12)^{j k l}$ & 5 & $2.25(0.05)^{j}$ & $1.91(0.02)^{1}$ & $1-2$ & $1.88(0.04)^{j k}$ & $30.84(1.98)$ abcde \\
\hline Cascona & $3.48(0.22)^{a}$ & 5 & $1.45(0.04)^{\mathrm{a}}$ & $1.44(0.05) \mathrm{g}$ & $1-2$ & $1.51(0.02)^{c}$ & $28.12(2.11)^{\mathrm{a}}$ \\
\hline Cornicabra & $4.02(0.11)^{b}$ & 5 & $1.72(0.07)^{b}$ & $1.23(0.04)^{\mathrm{c}}$ & 1 & $1.73(0.03)$ ef & $30.87(2.64)$ abcde \\
\hline De Convite & $4.70(0.15)$ hij & 5 & $2.05(0.05)^{\text {efg }}$ & $1.71(0.02)^{\mathrm{ij}}$ & $1-2$ & $1.95(0.02)^{l m}$ & $39.38(1.08) \mathrm{g}$ \\
\hline Desmayo Largueta & $4.31(0.13)$ cde & 5 & $2.02(0.06)$ ef & $1.45(0.05)^{\mathrm{g}}$ & $1-2$ & $1.65(0.04)^{d}$ & $29.51(2.06)^{a b c}$ \\
\hline Desmayo Rojo & $4.23(0.16)^{b c d}$ & 5 & $2.14(0.04)$ hi & $1.17(0.04)^{b}$ & $1-3$ & $1.79(0.03) \mathrm{gh}$ & $28.62(2.35)^{a}$ \\
\hline Esperanza & $4.26(0.14)$ bcde & 5 & $2.15(0.05)$ hi & $1.18(0.02)^{b}$ & $1-3$ & $1.81(0.03) \mathrm{hi}$ & $28.17(2.54)^{a}$ \\
\hline Gorda José & $5.03(0.21)^{\mathrm{kl}}$ & 5 & $2.25(0.06)^{j}$ & $2.04(0.03)^{m}$ & 1 & $1.83(0.03) \mathrm{hi}$ & $41.28(2.64)^{g}$ \\
\hline Marcelina & $5.11(0.17)^{1}$ & 5 & $2.39(0.05)^{k}$ & $1.63(0.05)^{h}$ & 1 & $1.76(0.04) \mathrm{fg}$ & $29.28(0.82)^{a b}$ \\
\hline Marcona & $4.62(0.20)$ ghi & 5 & $2.12(0.04)$ gh & $1.35(0.04)^{\mathrm{f}}$ & 1 & $1.48(0.02)^{b c}$ & $28.64(1.71)^{\mathrm{a}}$ \\
\hline Molar de Chavés & $4.60(0.18)$ fghi & 5 & $2.10(0.05)$ fgh & $1.32(0.03)^{\text {ef }}$ & 1 & $1.45(0.03)^{b}$ & $29.64(1.99) a b c$ \\
\hline Mollar de Arribes & $4.81(0.17))^{\mathrm{ijk}}$ & 5 & $2.22(0.08) \mathrm{ij}$ & $1.75(0.03) \mathrm{j}$ & 1 & $1.71(0.05)^{\mathrm{e}}$ & $34.02(1.82){ }^{\text {ef }}$ \\
\hline $\begin{array}{l}\text { Pestaneta de } \\
\text { Bragança }\end{array}$ & $4.16(0.19) \mathrm{bcd}$ & $5-6$ & $1.79(0.05)^{b}$ & $1.16(0.04)^{b}$ & 1 & $1.84(0.03)^{\mathrm{ij}}$ & $28.09(1.48)^{a}$ \\
\hline Peta & $5.51(0.17)^{\mathrm{m}}$ & 5 & $2.49(0.05)^{1}$ & $1.70(0.02)^{\mathrm{i}}$ & $1-2$ & $1.92(0.03) \mathrm{kl}$ & $31.02(1.49)$ abcdef \\
\hline Picuda & $4.52(0.17)$ efgh & $5-6$ & $1.99(0.06)$ de & $1.28(0.04)$ de & 1 & $1.45(0.04)^{b}$ & $53.64(3.02)^{h}$ \\
\hline Portuguesa & $5.02(0.22) \mathrm{kl}$ & 5 & $2.21(0.07)^{i j}$ & $1.86(0.05)^{k}$ & 1 & $2.05(0.02)^{n}$ & $33.05(2.64)$ def \\
\hline Recia & $4.99(0.14)^{\mathrm{kl}}$ & 5 & $2.25(0.03)^{j}$ & $1.62(0.08)^{h}$ & 1 & $1.98(0.02)^{\mathrm{m}}$ & $34.21(2.19)^{f}$ \\
\hline Redondilla & $4.31(0.12)^{\mathrm{cde}}$ & 5 & $1.93(0.02)^{\mathrm{cd}}$ & $1.23(0.02)^{c}$ & 1 & $1.89(0.04)^{k}$ & $29.15(1.43)^{\mathrm{ab}}$ \\
\hline Valenciana & $4.72(0.11)$ hij & 5 & $2.02(0.05)^{\text {ef }}$ & $1.71(0.03)^{\mathrm{ij}}$ & 1 & $1.95(0.03) \mathrm{lm}$ & $32.64(0.97)^{\text {cdef }}$ \\
\hline Verdeal & $4.18(0.16)^{b c d}$ & 5 & $1.80(0.04)^{b}$ & $1.35(0.04)^{f}$ & 1 & $1.75(0.03)^{\text {efg }}$ & $30.56(1.99)^{\mathrm{abcd}}$ \\
\hline Verdinal & $4.11(0.10) b c$ & 5 & $1.78(0.03)^{b}$ & $1.31(0.04)^{\text {def }}$ & 1 & $1.72(0.04)^{\text {ef }}$ & $30.67(1.35)^{\mathrm{abcd}}$ \\
\hline
\end{tabular}

ANOVA, analysis of variance; ${ }^{\text {a-n }}$ Different letters in the same column indicate statistically significant differences between cultivars at the $95 \%$ confidence level. 


\subsection{Leaves}

Leaf parameters are summarized in Table 2. Petiole length varied from 1.46 to $3.40 \mathrm{~cm}$, the cultivars with the shortest and the longest leaf peduncles being "Cornicabra" and "Mollar de Arribes", respectively. This last cultivar also showed the highest leaf blade length. At the opposite end for this parameter was the "Bravía" genotype. Its leaves were also the narrowest $(2.07 \mathrm{~cm})$. The length/width ratio of the leaf blades ranged from $3.76 \mathrm{~cm}$ to $7.91 \mathrm{~cm}$. "Valenciana", "Desmayo Rojo" and "Esperanza" were the cultivars with the highest ratios (5.96, 6.54 and 7.91, respectively). The other ratios calculated (petiole/leaf blade length) had values of around 0.22. The leaf glands showed green or reddish anthocyanin colorations.

\subsection{Fruits}

Fruit agromorphological parameters are shown in Table 3. Regarding dry fruit, "Gorda José" was the local cultivar that had the largest size and weight parameters, at $4.98 \mathrm{~cm}$ length, $3.41 \mathrm{~cm}$ width, $2.49 \mathrm{~cm}$ thickness and $16.35 \mathrm{~g}$ mass. Its nuts had geometric mean diameters close to $3.50 \mathrm{~cm}$ and volume and surface area values of around $15.64 \mathrm{~cm}^{3}$ and $38.02 \mathrm{~cm}^{2}$, respectively. At the other extreme was the cultivar "Bravía", with mean dry fruit dimensions of $2.82 \mathrm{~cm}$ (length), $1.82 \mathrm{~cm}$ (width) and $1.31 \mathrm{~cm}$ (thickness) and a mean mass value of about $2.70 \mathrm{~g}$. Its nuts also had the lowest values of volume $\left(2.42 \mathrm{~cm}^{3}\right)$ and surface area $\left(11.17 \mathrm{~cm}^{2}\right)$. Nut sphericity ranged from 60.31 to $79.00 \%$, the cultivars with the longest and roundest fruits being "Cornicabra" and "Marcona", respectively. It is also important to point out the relevant differences recorded for this parameter between the two "Mollar" cultivars. The "Molar de Arribes" genotype showed more elongated nuts than "Molar de Chavés" (64.22 and $72.29 \%$, respectively). With respect to shell hardness, high resistance to cracking was observed for all the cultivars studied, except for "Desmayo Rojo", "Mollar" and "De Convite". This latter cultivar has dry fruit which can easily be opened by birds. In relation to kernels, "Marcelina" was the traditional cultivar that had the largest size and weight parameters at $2.52 \mathrm{~cm}$ length, $1.94 \mathrm{~cm}$ width, $0.90 \mathrm{~cm}$ thickness and $2.11 \mathrm{~g}$ mass. Its kernels recorded geometric mean diameters close to $1.63 \mathrm{~cm}$ and volume and surface area values of around $1.56 \mathrm{~cm}^{3}$ and $8.41 \mathrm{~cm}^{2}$, respectively. With respect to sphericity, the kernels of this last cultivar were, together with those of the cultivars "Marcona" and "Agapitina", the roundest (values close to $65 \%$ ). The "Marcelina" genotype also recorded low values of double kernels (3\%). This tendency to produce only a single, well-formed kernel is a highly desirable cultivar trait. On the other hand, "Desmayo Largueta", "De Convite", "Cascona", "Cascafina", "Peta", "Esperanza" and "Desmayo Rojo" registered double kernel values higher than 14\%. With respect to the kernel/dry fruit mass ratio, "Cascafina" and "De Convite" were the cultivars with the highest yield values (0.34). It could be interesting to use these cultivars in future almond breeding programs. With respect to taste, all the cultivars were sweet except for the "Bravía" and "Recia" genotypes. 
Table 2. Means, standard deviations and ANOVA analyses for some leaf parameters in almond cultivars.

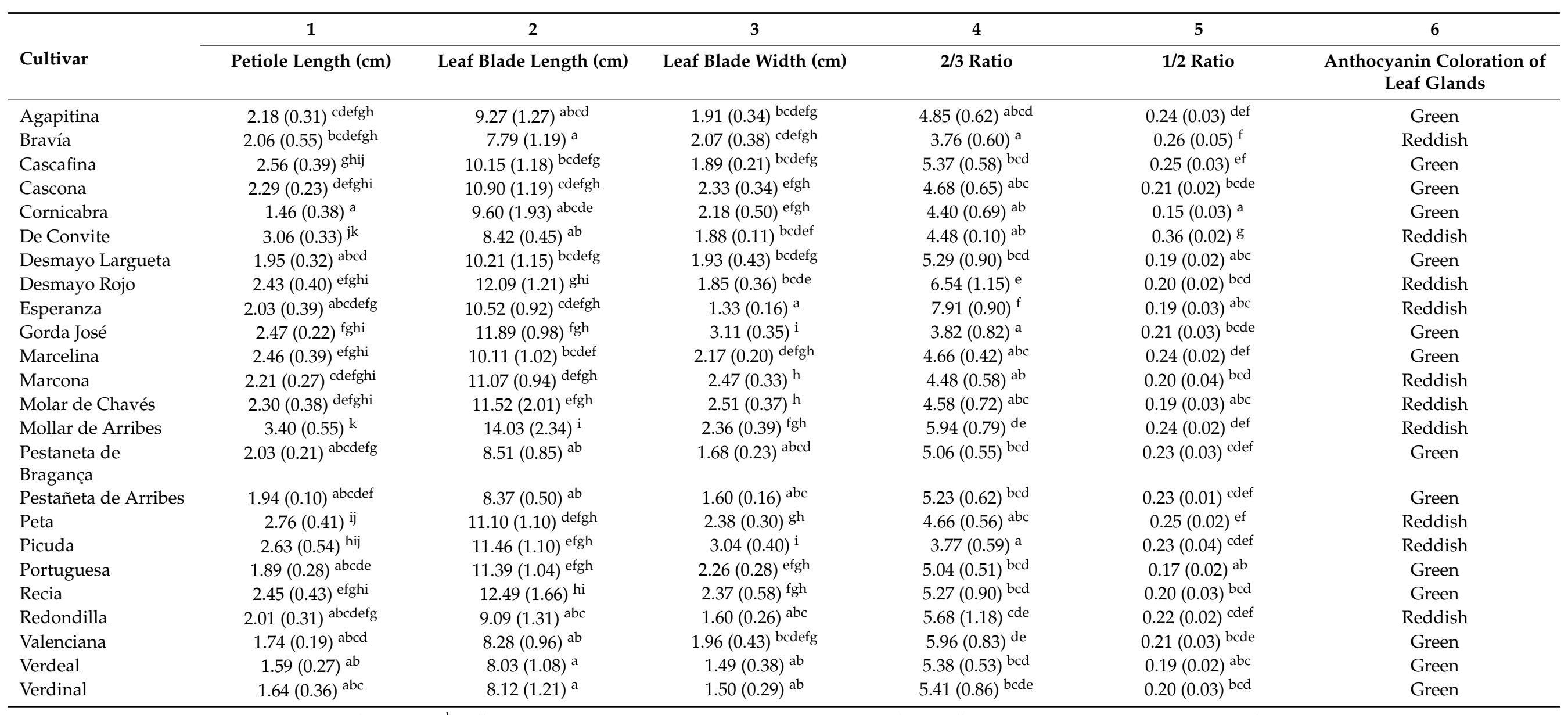

ANOVA, analysis of variance; ${ }^{a-k}$ Different letters in the same column indicate statistically significant differences between cultivars at the $95 \%$ confidence level. 
Table 3. Means, standard deviations and ANOVA analyses for some fruit parameters in almond cultivars.

\begin{tabular}{|c|c|c|c|c|c|c|}
\hline \multirow{2}{*}{ Cultivar } & \multicolumn{6}{|c|}{ Nut } \\
\hline & Weight (g) & Length $(\mathrm{cm})$ & Width $(\mathrm{cm})$ & Thickness (cm) & Volume $\left(\mathrm{cm}^{3}\right)$ & Geometric Mean Diameter (mm) \\
\hline Agapitina & $6.49(1.31)^{\mathrm{fg}}$ & $3.10(0.25)^{a b c}$ & $2.60(0.15)^{e f g}$ & $1.64(0.17)^{\mathrm{cd}}$ & $5.18(1.02)^{\text {bcdef }}$ & $23.62(2.16)$ bcd \\
\hline Bravía & $2.72(0.37)^{\mathrm{a}}$ & $2.82(0.15)^{\mathrm{a}}$ & $1.82(0.28)^{\mathrm{a}}$ & $1.31(0.06)^{\mathrm{a}}$ & $2.42(0.67)^{\mathrm{a}}$ & $18.86(1.94)^{\mathrm{a}}$ \\
\hline Cascafina & $3.17(2.02)^{a b}$ & $3.38(0.19)$ bcdefgh & $2.42(0.15)$ cde & $1.56(0.08) b c$ & $4.68(1.15)$ bcde & $23.34(2.00) \mathrm{bcd}$ \\
\hline Cascona & $5.50(1.15)^{\operatorname{cdefg}}$ & $3.45(0.27)$ cdefghi & $2.40(0.22)$ cde & $1.61(0.14)^{b c d}$ & $4.88(1.09)^{\text {bcdef }}$ & $23.69(2.08) \mathrm{bcd}$ \\
\hline Cornicabra & $5.11(1.28)$ cdef & $3.83(0.45)^{\mathrm{ij}}$ & $2.07(0.17) a b$ & $1.56(0.08) \mathrm{bc}$ & $4.23(0.98))^{b c}$ & $23.10(1.96) b c$ \\
\hline De Convite & $3.86(0.30)^{a b c}$ & $3.70(0.09)$ ghij & $2.37(0.21)$ bcde & $1.45(0.10)^{a b}$ & $4.44(1.12) \mathrm{bcd}$ & $23.32(2.28) \mathrm{bcd}$ \\
\hline Desmayo Rojo & $5.00(0.77)^{\text {cdef }}$ & $3.24(0.18)$ bcdef & $2.46(0.13){ }^{\text {cde }}$ & $1.64(0.14)^{\mathrm{cd}}$ & $4.95(1.22)$ bcdef & $23.53(2.07)^{b c d}$ \\
\hline Esperanza & $4.80(0.59)$ bcde & $3.38(0.08)$ bcdefgh & $2.52(0.13)$ de & $1.64(0.11)^{\mathrm{cd}}$ & $5.22(1.41)$ bcdef & $24.08(1.96)^{b c d}$ \\
\hline Gorda José & $16.35(0.87)^{\mathrm{j}}$ & $4.98(0.23)^{\mathrm{k}}$ & $3.41(0.15)^{j}$ & $2.49(0.11)^{g}$ & $15.64(3.83)^{j}$ & $34.79(2.41)^{f}$ \\
\hline Marcelina & $9.14(0.52)^{\mathrm{i}}$ & $3.48(0.15)$ cdefghi & $2.88(0.10)$ ghi & $1.85(0.10)$ ef & $7.26(1.42) \mathrm{hi}$ & $26.44(2.17) \mathrm{de}$ \\
\hline Marcona & 5.77 (1.15) defg & $3.13(0.20)^{a b c d}$ & $2.65(0.20)$ efg & $1.83(0.16)$ ef & $6.13(1.00)$ efgh & 24.73 (2.09) bcde \\
\hline Molar de Chavés & $5.65(0.99)$ defg & $3.30(0.39)$ bcdefg & $2.54(0.20)$ ef & $1.62(0.14)^{\mathrm{cd}}$ & $5.13(1.08)^{\text {bcdef }}$ & $23.85(1.99) \mathrm{bcd}$ \\
\hline Mollar de Arribes & $5.43(0.90)$ cdefg & $3.79(0.60)$ hij & $2.40(0.22)^{\text {cde }}$ & $1.59(0.12) b c$ & $5.10(1.14)^{\text {bcdef }}$ & $24.34(1.86)^{b c d}$ \\
\hline $\begin{array}{l}\text { Pestaneta de } \\
\text { Bragança }\end{array}$ & $6.87(1.01)^{g h}$ & $3.20(0.21)$ abcdef & $2.64(0.19)$ efg & $1.64(0.10)^{\mathrm{cd}}$ & $5.37(1.20)$ cdefg & $23.99(1.99)^{b c d}$ \\
\hline Peta & $8.22(1.04) \mathrm{hi}$ & $4.09(0.17)^{j}$ & $2.67(0.12)^{\text {efg }}$ & $1.99(0.12)^{f}$ & $7.92(1.52)^{\mathrm{i}}$ & $27.87(2.30)^{\mathrm{e}}$ \\
\hline Picuda & $4.90(0.85)^{\text {cdef }}$ & $3.54(0.21)$ defghi & $2.85(0.20)$ fgh & $1.60(0.06)^{b c d}$ & $6.05(1.29)$ defgh & $25.24(2.04)$ cde \\
\hline Portuguesa & $6.51(0.92)^{\mathrm{fg}}$ & $3.61(0.22)$ fghi & $2.68(0.16)^{\text {efg }}$ & $1.76(0.09)$ de & $6.37(1.04)^{\text {fghi }}$ & $25.70(2.15)$ cde \\
\hline Recia & $5.43(0.69)^{\operatorname{cdefg}}$ & $2.98(0.24)^{a b}$ & $2.19(0.22) b c$ & $1.62(0.26)^{\mathrm{cd}}$ & $4.04(0.97) a b c$ & $21.93(1.78)^{a b}$ \\
\hline Redondilla & $6.18(1.81)^{\text {efg }}$ & $3.17(0.47)$ abcde & $2.50(0.33)^{\text {cde }}$ & $1.72(0.15)$ cde & $5.30(1.11)^{\text {cdefg }}$ & $23.86(1.96)^{b c d}$ \\
\hline Valenciana & $6.51(0.98)^{f g}$ & $3.30(0.24)$ bcdefg & $2.57(0.18){ }^{\text {efg }}$ & $1.85(0.12) e^{\text {ef }}$ & $6.13(1.26)$ efgh & $25.01(2.07)$ bcde \\
\hline Verdeal & $6.23(1.02)^{\text {efg }}$ & $3.40(0.20)$ cdefgh & $3.17(0.25)$ hij & $1.63(0.11)^{\mathrm{cd}}$ & $6.90(1.47)$ ghi & $25.97(2.13)$ cde \\
\hline Verdinal & $6.38(0.78)$ efg & $3.42(0.16)$ cdefghi & $3.20(0.28)^{\mathrm{ij}}$ & $1.65(0.09)^{\mathrm{cd}}$ & $7.11(1.33)$ hi & $26.21(2.24)$ cde \\
\hline
\end{tabular}


Table 3. Cont.

\begin{tabular}{|c|c|c|c|c|c|c|}
\hline \multirow{2}{*}{ Cultivar } & \multicolumn{3}{|c|}{ Nut } & \multicolumn{3}{|c|}{ Kernel } \\
\hline & Sphericity (\%) & Surface Area $\left(\mathrm{cm}^{2}\right)$ & Shell Hardness & Weight (g) & Length (cm) & Width (cm) \\
\hline Agapitina & $76.19(6.22)$ ef & $17.52(1.82)^{b c d}$ & Hard & $1.47(0.23)$ ef & $2.26(0.22)^{a b}$ & $1.69(0.22)^{\mathrm{ijk}}$ \\
\hline Bravía & $66.87(5.16)$ abcde & $11.17(2.03)^{\mathrm{a}}$ & Hard & $0.89(0.14)^{a}$ & $2.26(0.11)^{\mathrm{ab}}$ & $1.10(0.18)^{\mathrm{a}}$ \\
\hline Cascafina & $69.05(5.62)$ abcde & $17.11(1.96)^{b c}$ & Hard & $1.09(0.14)^{a b c d}$ & $2.43(0.14)$ bcdef & $1.53(0.07)$ fghi \\
\hline Cascona & $68.66(5.47)$ abcde & $17.63(2.15)^{b c d}$ & Hard & $1.40(0.28)$ def & $2.43(0.17)$ bcdef & $1.48(0.13)$ defg \\
\hline Cornicabra & $60.31(4.98)^{\mathrm{a}}$ & $16.76(1.85)^{b c}$ & Hard & $1.27(0.14)^{\text {cdef }}$ & $2.65(0.23)$ def & $1.35(0.20)$ bcde \\
\hline De Convite & $63.02(5.29)^{a b c}$ & $17.08(2.31) b c$ & Soft & $1.35(0.12)$ cdef & $2.67(0.12)$ ef & $1.50(0.06)$ efg \\
\hline Desmayo Rojo & $72.62(6.00)^{\text {cdef }}$ & $17.39(1.95)^{b c}$ & Intermediate & $1.18(0.14)^{\text {abcdef }}$ & $2.30(0.15)^{a b c}$ & $1.33(0.09)^{b c d}$ \\
\hline Esperanza & $71.25(6.43)$ bcdef & $18.21(2.04)$ bcde & Hard & $0.92(0.07)^{\mathrm{ab}}$ & $2.23(0.06)^{a b}$ & $1.30(0.08)^{b c}$ \\
\hline Gorda José & $69.85(5.48)$ abcdef & $38.02(3.52) \mathrm{h}$ & Hard & $1.94(0.18)^{g}$ & $2.61(0.18)^{\text {cdef }}$ & $1.80(0.11)^{\mathrm{kl}}$ \\
\hline Marcelina & $75.97(6.11)^{\text {ef }}$ & $21.96(2.01)^{\mathrm{fg}}$ & Hard & $2.11(0.23)^{g}$ & $2.52(0.22)$ bcdef & $1.94(0.05)^{1}$ \\
\hline Marcona & $79.00(5.87)^{\mathrm{f}}$ & $19.21(1.93)^{\text {cdefg }}$ & Hard & $1.30(0.14)^{\text {cdef }}$ & $2.08(0.10)^{\mathrm{a}}$ & $1.58(0.14)$ ghi \\
\hline Molar de Chavés & $72.29(5.00)^{\text {cdef }}$ & $17.87(1.87) \mathrm{bcd}$ & Intermediate & $1.33(0.16)^{\text {cdef }}$ & $2.32(0.21) a b c$ & $1.55(0.16)$ fghi \\
\hline Mollar de Arribes & $64.22(5.42)^{a b c d}$ & $18.61(2.26)^{\text {cdef }}$ & Intermediate & $1.39(0.13)$ def & $2.51(0.24)$ bcdef & $1.52(0.14)$ fgh \\
\hline $\begin{array}{l}\text { Pestaneta de } \\
\text { Bragança }\end{array}$ & $74.98(6.31)^{\text {ef }}$ & $18.08(2.38)$ bcde & Hard & $1.37(0.11)^{\text {cdef }}$ & $2.32(0.16)^{a b c}$ & $1.76(0.09))^{j k}$ \\
\hline Peta & $68.14(5.87)$ abcde & $24.40(2.49)^{g}$ & Hard & $1.39(0.22)$ def & $2.72(0.15)^{f}$ & $1.67(0.09)^{h i j k}$ \\
\hline Picuda & $71.29(6.01)$ bcdef & 20.01 (2.09) cdefg & Hard & $1.21(0.11)^{\text {abcdef }}$ & $2.25(0.15)^{a b}$ & $1.50(0.08)$ efg \\
\hline Portuguesa & $71.19(5.99)$ bcdef & $20.74(2.14)$ defg & Hard & $1.48(0.22)^{f}$ & $2.55(0.13)$ bcdef & $1.62(0.08)$ ghij \\
\hline Recia & $73.59(6.14)$ def & $15.10(1.52)^{b}$ & Hard & $1.23(0.17)$ bcdef & $2.29(0.13)^{a b c}$ & $1.40(0.08)$ cdef \\
\hline Redondilla & $75.26(5.62)$ ef $^{-1}$ & $17.07(1.76)^{b c}$ & Hard & $1.19(0.21)^{\text {abcdef }}$ & $2.34(0.14)^{\mathrm{abad}}$ & $1.55(0.16)$ fghi \\
\hline Valenciana & $75.78(5.76){ }^{\text {ef }}$ & 19.65 (1.64) cdefg & Hard & $1.15(0.19)$ abcde & $2.24(0.14)^{a b}$ & 1.55 (0.09) fghi \\
\hline Verdeal & $76.38(6.20))^{\text {ef }}$ & $21.18(2.37)$ defg & Hard & $1.30(0.17)^{\text {cdef }}$ & $2.39(0.17)^{\text {abcde }}$ & $1.46(0.11)^{\text {cdefg }}$ \\
\hline Verdinal & $76.63(6.07))^{e f}$ & $21.58(2.43)$ efg & Hard & $1.31(0.21)^{\text {cdef }}$ & $2.41(0.20)$ bcdef & $1.47(0.13)$ defg \\
\hline
\end{tabular}


Table 3. Cont.

\begin{tabular}{|c|c|c|c|c|c|c|c|}
\hline \multirow[b]{2}{*}{ Cultivar } & \multicolumn{7}{|c|}{ Kernel } \\
\hline & Thickness (cm) & Volume $\left(\mathrm{cm}^{3}\right)$ & $\begin{array}{c}\text { Geometric Mean } \\
\text { Diameter (mm) }\end{array}$ & Sphericity (\%) & Surface Area $\left(\mathrm{cm}^{2}\right)$ & Doubles (\%) & Taste \\
\hline Agapitina & $0.85(0.08)^{b c}$ & $1.16(0.21)^{\mathrm{c}}$ & $14.80(1.02)^{\mathrm{cd}}$ & $65.49(4.94) \mathrm{hi}$ & $6.88(0.62)$ de & 2 & Sweet \\
\hline Bravía & $0.84(0.06)^{a b c}$ & $0.69(0.23)^{\mathrm{a}}$ & $12.77(1.11)^{\mathrm{a}}$ & $56.54(3.21) \mathrm{bcd}$ & $5.12(0.59)^{\mathrm{ab}}$ & 6 & Bitter \\
\hline Cascafina & $0.73(0.06)^{a b}$ & $0.91(0.20)^{a b c}$ & $13.94(0.96)^{a b c}$ & $57.38(4.89)$ bcdef & $6.10(0.70)^{a b c d}$ & 17 & Sweet \\
\hline Cascona & $0.85(0.08)^{b c}$ & $1.04(0.19) b c$ & $14.50(1.20) b c$ & $59.70(3.93)$ cdefghi & $6.60(0.71)^{a b c d}$ & 16 & Sweet \\
\hline Cornicabra & $0.80(0.06)^{a b c}$ & $0.93(0.20)^{a b c}$ & $14.19(0.99)^{a b c}$ & $53.55(3.65) a b c$ & $6.32(0.68)^{\text {abcd }}$ & 3 & Sweet \\
\hline De Convite & $0.70(0.06)^{a b}$ & $0.91(0.22){ }^{a b c}$ & $14.09(1.14)^{a b c}$ & $52.79(4.07)^{\mathrm{ab}}$ & $6.23(0.67)^{a b c d}$ & 15 & Sweet \\
\hline Desmayo Largueta & $0.68(0.09)^{\mathrm{a}}$ & $0.68(0.18)^{\mathrm{a}}$ & $12.88(0.90)^{\mathrm{ab}}$ & $49.54(4.62)^{\mathrm{a}}$ & $5.21(0.62){ }^{a b c}$ & 14 & Sweet \\
\hline Desmayo Rojo & $0.80(0.06)^{a b c}$ & $0.83(0.21)^{a b}$ & $13.47(0.94)^{a b c}$ & 58.57 (3.94) bcdefg & $5.70(0.66)^{a b c d}$ & 21 & Sweet \\
\hline Esperanza & $0.70(0.07)^{\mathrm{ab}}$ & $0.68(0.19)^{\mathrm{a}}$ & $12.66(1.03)^{a}$ & $56.77(3.82)$ bcde & $5.03(0.60)^{\mathrm{a}}$ & 20 & Sweet \\
\hline Gorda José & $0.70(0.04)^{\mathrm{ab}}$ & $1.10(0.23)^{b c}$ & $14.86(1.00)^{\mathrm{cd}}$ & $56.95(3.81)^{\text {bcde }}$ & $6.93(0.72) \mathrm{de}$ & 0 & Sweet \\
\hline Marcelina & $0.90(0.06)^{\mathrm{c}}$ & $1.56(0.21)^{\mathrm{d}}$ & $16.37(1.28)^{d}$ & $64.99(4.83)$ ghi & $8.41(0.78)^{\mathrm{e}}$ & 3 & Sweet \\
\hline Marcona & $0.78(0.06)^{a b c}$ & $0.92(0.22)^{a b c}$ & $13.68(0.97)^{a b c}$ & $65.77(5.02)^{\mathrm{i}}$ & $5.87(0.70)^{a b c d}$ & 4 & Sweet \\
\hline Molar de Chavés & $0.80(0.07)^{a b c}$ & $0.99(0.18)^{a b c}$ & $14.22(1.09)^{a b c}$ & $61.30(4.15)$ defghi & $6.35(0.75)^{\text {abcd }}$ & 7 & Sweet \\
\hline Mollar de Arribes & $0.82(0.10)^{a b c}$ & $1.05(0.20) b c$ & $14.61(1.04)^{\mathrm{c}}$ & $58.24(4.41)$ bcdef & $6.70(0.69) \mathrm{bcd}$ & 5 & Sweet \\
\hline $\begin{array}{l}\text { Pestaneta de } \\
\text { Bragança }\end{array}$ & $0.80(0.06)^{a b c}$ & $1.15(0.23) b c$ & $14.83(1.11)^{\mathrm{cd}}$ & $63.92(4.87){ }^{\text {efghi }}$ & $6.90(0.71)^{\text {cde }}$ & 3 & Sweet \\
\hline Pestañeta de Arribes & $0.80(0.07)^{a b c}$ & $1.13(0.19)^{b c}$ & $14.76(1.13)^{\mathrm{cd}}$ & $64.17(5.06)$ fghi & $6.84(0.70)$ de & 4 & Sweet \\
\hline Peta & $0.69(0.09)^{\mathrm{ab}}$ & $1.02(0.21)^{b c}$ & $14.62(0.98)^{c}$ & $53.78(3.94)^{a b c}$ & $6.71(0.73)^{b c d}$ & 17 & Sweet \\
\hline Picuda & $0.85(0.06)^{b c}$ & $1.00(0.22)^{a b c}$ & $14.20(1.17)^{a b c}$ & $63.12(4.08)$ defghi & $6.33(0.69)^{\mathrm{abad}}$ & 4 & Sweet \\
\hline Portuguesa & $0.79(0.05)^{a b c}$ & $1.10(0.20)^{b c}$ & $14.82(1.20)^{\mathrm{cd}}$ & $58.14(4.61)$ bcdef & $6.89(0.72) \mathrm{de}$ & 2 & Sweet \\
\hline Recia & $0.80(0.08)^{a b c}$ & $0.87(0.23)^{a b c}$ & $13.68(0.99)^{a b c}$ & $59.75(4.19)$ cdefghi & $5.87(0.67)^{a b c d}$ & 8 & Intermediate \\
\hline Redondilla & $0.79(0.06)^{a b c}$ & $0.98(0.21)^{a b c}$ & $14.19(1.02)^{a b c}$ & $60.67(3.76)$ defghi & $6.32(0.70)^{a b c d}$ & 4 & Sweet \\
\hline Valenciana & $0.70(0.08)^{a b}$ & $0.83(0.22)^{a b}$ & $13.44(0.97)^{a b c}$ & $60.00(4.99)$ cdefghi & $5.67(0.68)^{\text {abcd }}$ & 3 & Sweet \\
\hline Verdeal & $0.79(0.06)^{a b c}$ & $0.93(0.19)^{a b c}$ & $14.01(1.06)^{a b c}$ & $58.64(4.52)$ bcdefgh & $6.16(0.73)$ abcd & 5 & Sweet \\
\hline Verdinal & $0.80(0.05)^{a b c}$ & $0.96(0.21)^{a b c}$ & $14.14(1.10)^{a b c}$ & $58.70(3.84)$ bcdefgh & $6.28(0.69)^{\mathrm{abcd}}$ & 5 & Sweet \\
\hline
\end{tabular}


Fruit chemical parameters are summarized in Table 4. The kernel dry weight value was quite similar in all the cases (95.54-96.56\%). "Mollar de Arribes" was the cultivar with the lowest water level in kernels. Greater differences were recorded for the rest of the chemical parameters. Lipid content varied from $48.82 \%$ to $56.80 \%$, "Mollar de Arribes", "Cascafina" and "Peta" being the cultivars with the most oleaginous kernels. Lipids content is highly correlated with energy levels $(\mathrm{r}=0.95)$. On the other hand, it is also important to note that the "Molar de Chavés" cultivar, the other genotype called "Mollar" by the local growers, recorded a very low lipids level, close to $49 \%$. Protein content ranged between $15.54 \%$ and $23.39 \%$, "Peta" and "Esperanza" being the cultivars with the lowest and the highest values, respectively. It can be observed that protein content is inversely correlated with the lipid fraction $(\mathrm{r}=-0.87)$. Dietary fiber content varied from 14.03 to $17.99 \%$. "Picuda", "Gorda José" and "Peta" were the cultivars that had the highest values. Carbohydrates content varied from $2.86 \%$ to $4.82 \%$, "Recia", "Desmayo Rojo" and "Esperanza" being the cultivars with the highest levels. Finally, ash content ranged from $3.02 \%$ to $4.17 \%$. It can be observed that the results were very similar for all the almond cultivars.

\subsection{Vegetative Tree Habits}

Very different vegetative habits were observed, ranging from very upright or upright to completely drooping. The habit of the "Agapitina" cultivar was between upright and very upright. On the opposite side, "Desmayo Largueta', "Verdeal" and "Verdinal" were the only almond genotypes that had drooping growth habits. The rest of the cultivars showed vegetative habits between medium and spreading. This was the case for "Mollar de Arribes", "Pestaneta de Bragança", "Pestañeta de Arribes" and "Valenciana" (mediumupright); "Cascafina", "Desmayo Rojo", "Esperanza", "Mollar de Arribes" and "Peta" (medium); "Bravía" (medium-spreading); "Cascona", "De Convite”, "Gorda José" and "Portuguesa" (spreading); and "Cornicabra", "Marcelina”, "Marcona", "Picuda", "Recia” and "Redondilla" (spreading-drooping). Finally, it is also important to note that the two genotypes called "Mollar" by the local growers, "Molar de Chavés" and "Mollar de Arribes", showed medium-upright and medium growth habits, respectively.

\subsection{Statistical Analyses}

Principal component analysis (PCA) was used to identify the traits with the highest variation between cultivars and the greatest impact on separation of them in the dataset [45]. PCA results based on flower, leaf, fruit and tree traits showed that more than $54 \%$ of the variability observed was explained by the first three components (PC1-PC3). The first component (PC1), accounting for $27.65 \%$ of the total variance, was influenced by nut weight and size parameters such as thickness, volume, geometric mean diameter and surface area. The second component (PC2) accounted for $15.27 \%$ of total variation and was mainly explained by leaf petiole length and nut and kernel sphericity. Finally, the third principal component (PC3), explaining $11.72 \%$ of total variation, was integrated by the kernel thickness and the lipid content. Figure 1 shows a scatterplot of the first two principal components (PCs) for the 24 traditional almond cultivars based on agromorphological and chemical characteristics. It can be observed that there was high variation between genotypes, indicating that the studied germplasm is a good gene pool candidate for breeding programs. 
Table 4. Means, standard deviations and ANOVA analyses for some parameters of nutritive composition in almond cultivars.

\begin{tabular}{|c|c|c|c|c|c|c|c|}
\hline Cultivar & Dry Weight (\%) & Lipids (\%) & Proteins (\%) & Dietary Fiber (\%) & Carbohydrates (\%) & Ash (\%) & Energy $(\mathrm{kcal} / 100 \mathrm{~g})$ \\
\hline Agapitina & $95.82(0.71)^{a b}$ & $52.76(2.06)^{\mathrm{cd}}$ & $20.81(0.92)^{\mathrm{cd}}$ & $15.03(0.96)^{a b}$ & $3.82(0.42)$ cde & $3.55(0.31)^{\text {bcdef }}$ & $573.28(15.07)$ bcdef \\
\hline Bravía & $95.69(0.86)^{a b}$ & $50.87(2.92)^{a b c}$ & $23.06(1.04)^{\text {efg }}$ & $14.84(1.13)^{a b}$ & $4.04(0.58)$ cdef & $3.03(0.29)^{\mathrm{a}}$ & $566.15(20.11)^{a b c d}$ \\
\hline Cascafina & $96.14(0.79)^{a b}$ & $56.13(2.00) \mathrm{fg}$ & $18.57(0.87)^{b}$ & $14.81(1.35)^{\mathrm{ab}}$ & $3.69(0.50)^{c}$ & $3.16(0.34)^{a b c d}$ & $593.83(12.79)$ ef \\
\hline Cascona & $95.67(0.69)^{a b}$ & $52.67(2.76)^{\mathrm{cd}}$ & $21.09(0.96)^{\mathrm{cd}}$ & $15.19(1.27)^{a b}$ & $3.97(0.52)$ cde & $3.09(0.39) a b c$ & $573.59(18.34)$ bcdef \\
\hline Cornicabra & $95.76(0.74)^{a b}$ & $52.94(3.01)^{\text {cdef }}$ & $20.75(0.83)^{\mathrm{cd}}$ & $14.78(1.76)^{a b}$ & $3.76(0.57)^{\mathrm{cd}}$ & $3.77(0.30)$ efghi & $574.26(13.92)$ bcdef \\
\hline De Convite & $96.33(0.77)^{a b}$ & $52.83(2.61)$ cde & $21.63(0.91)$ def & $14.97(0.92)^{a b}$ & $3.91(0.45)$ cde & $3.11(0.33)^{a b}$ & $577.59(14.67)^{\text {cdef }}$ \\
\hline Desmayo Largueta & $95.69(0.80)^{a b}$ & $50.77(2.42){ }^{a b c}$ & $21.64(0.94)$ def & $15.56(1.51)^{\mathrm{ab}}$ & $4.05(0.51)^{\text {cdef }}$ & $3.82(0.36)$ efghi & $559.49(16.82)^{a b c}$ \\
\hline Desmayo Rojo & $96.21(0.74)^{a b}$ & $52.83(2.38)$ cde & $21.02(1.02)^{\mathrm{cd}}$ & $14.33(1.10)^{\mathrm{a}}$ & $4.63(0.47){ }^{e f}$ & $3.55(0.29)$ bcdef & $577.95(11.30)$ bcdef \\
\hline Esperanza & $95.80(0.79)^{b}$ & $49.08(1.97)^{\mathrm{ab}}$ & $23.39(1.11)^{\mathrm{g}}$ & $15.07(1.60)^{\mathrm{ab}}$ & $4.56(0.49)$ def & $4.09(0.38)$ ghi & $553.28(17.83)^{\mathrm{ab}}$ \\
\hline Gorda José & $96.55(0.68)^{a b}$ & $50.26(2.49)^{a b c}$ & $21.64(0.99)$ def & $16.26(1.09)^{b}$ & $4.47(0.60){ }^{\text {cdef }}$ & $4.17(0.28)^{\mathrm{i}}$ & $556.50(14.12)^{a b c}$ \\
\hline Marcelina & $95.94(0.83)^{a b}$ & $52.52(2.61)^{\mathrm{cd}}$ & $20.95(0.86)^{\mathrm{cd}}$ & $14.74(1.54)^{a b}$ & $3.71(0.58)^{\mathrm{c}}$ & $4.12(0.33) \mathrm{hi}$ & $571.28(15.99)^{\text {abcde }}$ \\
\hline Marcona & $96.03(0.78)^{a b}$ & $52.47(2.46)^{\mathrm{cd}}$ & $21.16(0.94)^{\mathrm{cd}}$ & $15.03(1.49)^{a b}$ & $4.10(0.52){ }^{\text {cdef }}$ & $3.46(0.37)$ abcdef & $573.27(21.67)$ abcde \\
\hline Molar de Chavés & $95.54(0.84)^{a}$ & $49.91(1.95)^{a b c}$ & $23.21(0.97) \mathrm{fg}$ & $14.96(0.90)^{\mathrm{ab}}$ & $3.99(0.43)$ cde & $3.64(0.30)$ defgh & $557.63(17.22)^{a b c}$ \\
\hline Mollar de Arribes & $96.56(0.80)^{\mathrm{ab}}$ & $56.80(2.81)^{\mathrm{g}}$ & $19.67(0.87) b c$ & $15.08(1.82)^{a b}$ & $2.13(0.46)^{\mathrm{a}}$ & $3.02(0.28)^{a}$ & $598.28(10.65)^{f}$ \\
\hline $\begin{array}{l}\text { Pestaneta de } \\
\text { Bragança }\end{array}$ & $96.08(0.75)^{a b}$ & $52.73(3.02)^{\mathrm{cd}}$ & $20.73(0.85)^{\mathrm{cd}}$ & $15.36(1.74)^{a b}$ & $4.04(0.52){ }^{\text {cdef }}$ & $3.36(0.37)$ abcde & $573.49(18.34)$ bcdef \\
\hline Pestañeta de Arribes & $95.91(0.87)^{a b}$ & $52.97(2.43)$ cdef & $20.57(0.92)^{\mathrm{cd}}$ & $15.14(1.66)^{a b}$ & $3.80(0.44)^{\mathrm{cd}}$ & $3.64(0.33)$ defgh & $574.21(13.96){ }^{\text {bcdef }}$ \\
\hline Picuda & $95.59(0.69)^{\mathrm{a}}$ & $48.82(2.09)^{\mathrm{a}}$ & $23.35(0.90)^{g}$ & $15.64(0.98)^{a b}$ & $4.07(0.50){ }^{\text {cdef }}$ & $3.85(0.27)$ fghi & $548.78(12.83)^{\mathrm{a}}$ \\
\hline Portuguesa & $95.54(0.68)^{\mathrm{a}}$ & $50.06(2.31)^{a b c}$ & $23.33(1.00)^{\mathrm{g}}$ & $14.93(1.16)^{a b}$ & $3.83(0.53)$ cde & $3.53(0.35)^{\text {bcdef }}$ & $559.06(15.71)^{a b c}$ \\
\hline Recia & $95.96(0.81)^{a b}$ & $54.77(2.73)$ defg & $18.78(0.83)^{b}$ & $14.04(1.07)^{\mathrm{a}}$ & $4.82(0.56)^{f}$ & $3.79(0.37)$ efghi & $587.25(19.80))^{\text {def }}$ \\
\hline Redondilla & $95.94(0.75)^{\mathrm{ab}}$ & $51.86(2.64)^{\text {abcd }}$ & $21.56(1.06) \mathrm{de}$ & $14.82(0.93)^{a b}$ & $3.86(0.49)$ cde & $4.04(0.33)$ ghi & $568.18(11.82)^{a b c d}$ \\
\hline Valenciana & $96.23(0.70)^{a b}$ & $52.24(2.82) \mathrm{bcd}$ & $21.82(0.98)$ defg & $14.99(1.02)^{a b}$ & $3.71(0.47)^{c}$ & $3.61(0.40)^{\text {cdefg }}$ & $572.24(21.23)$ abcde \\
\hline Verdeal & $96.17(0.83)^{\mathrm{ab}}$ & $52.73(2.71)^{\mathrm{cd}}$ & $20.73(1.11)^{\mathrm{cd}}$ & $14.88(1.14)^{a b}$ & $3.93(0.60)^{\text {cde }}$ & $4.05(0.36)$ ghi & $573.09(16.79)$ bcdef \\
\hline Verdinal & $96.02(0.75)^{a b}$ & $53.05(2.00){ }^{\text {cdef }}$ & $22.14(0.94)$ defg & $14.03(0.91)^{\mathrm{a}}$ & $3.65(0.54) b c$ & $3.38(0.31)^{\text {abcdef }}$ & $580.41(14.27)^{\text {cdef }}$ \\
\hline
\end{tabular}




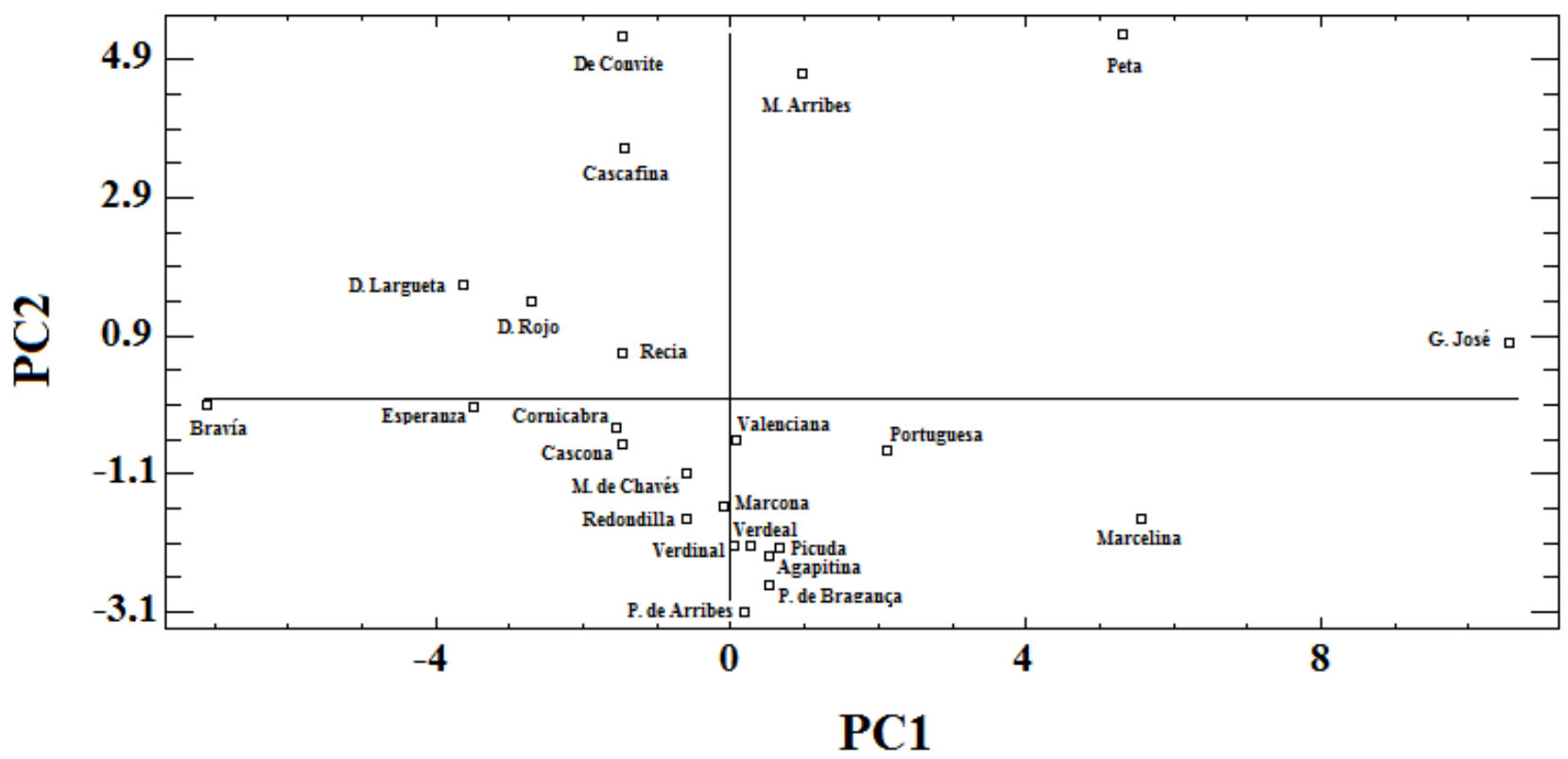

Figure 1. Scatterplot of the first two principal components (PCs) for the 24 traditional almond cultivars based on agromorphological and chemical characteristics.

Figure 2 shows a dendrogram of the relationships among the almond cultivars from the analysis of all the agromorphological and chemical parameters studied. It can be observed that the "Gorda José" cultivar showed the greatest differences compared to the rest of cultivars included in the study. Its nuts showed a large size in comparison with the others.

\section{0}

300

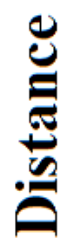

200

100

0

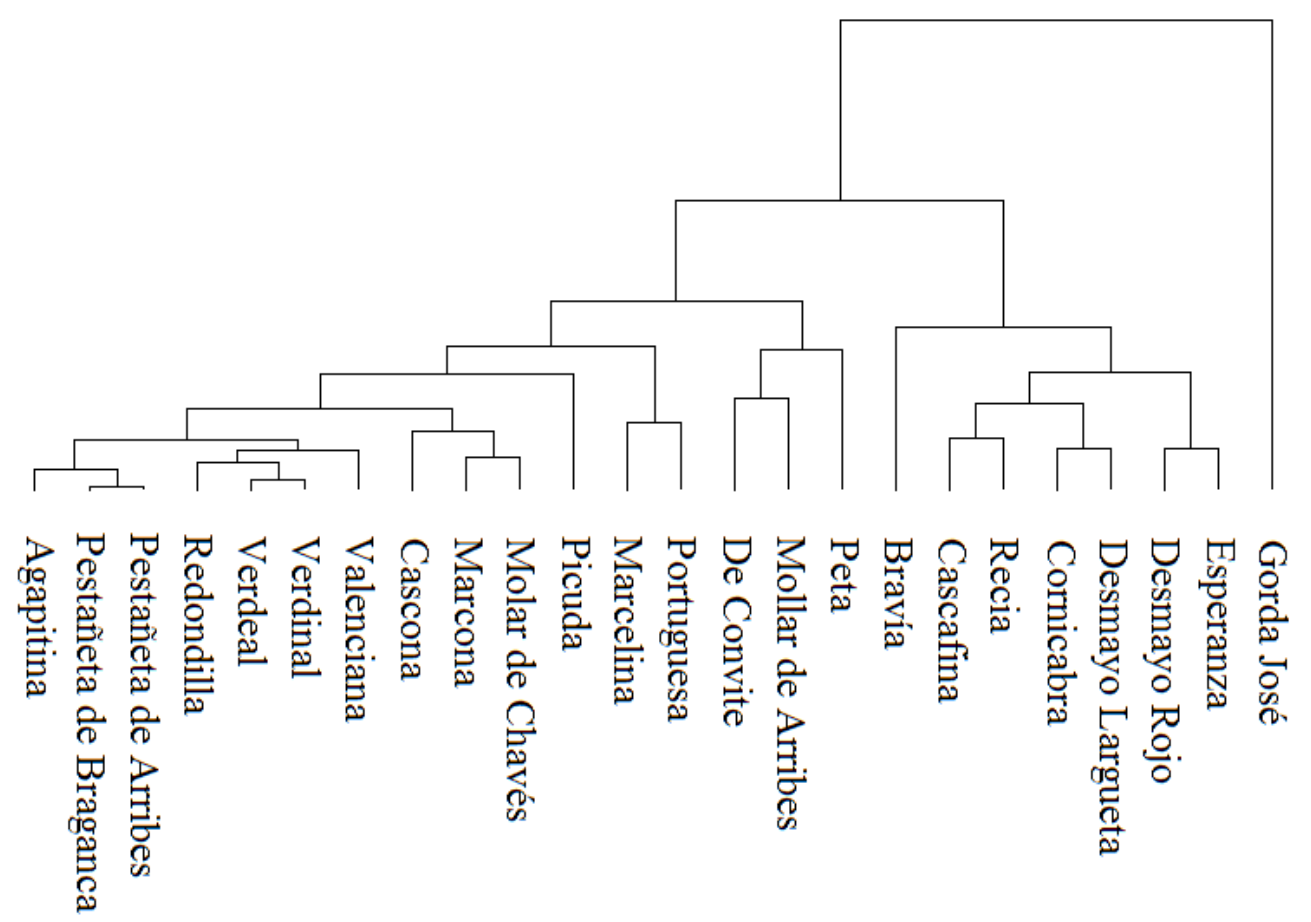

Figure 2. Dendrogram produced using the furthest neighbor method (Euclidean) from the agromorphological and chemical characteristics of traditional almond cultivars included in the study. 


\section{Discussion}

\subsection{Agromorphological and Chemical Characterization}

The traditional almond cultivars from the central-western Iberian Peninsula showed great variability from an agromorphological point of view. Many of them, despite being practically unknown in the scientific literature, presented very interesting productive characters. Some previous researchers have provided information about some of the cultivars included in the study ("Marcona" and "Desmayo Largueta").

With respect to the flowers, Socias i Company et al. [46] also observed that there are important variations among almond cultivars. They indicated that the flower size is frequently related to the nut size and that the numbers of petals and pistils can range in relation to the type flower. With regard to the stamens, they established that their number can oscillate between 20 and 30, but may reach 40 . In our study, a reduced number of cultivars showed six petals or two/three pistils and the number of stamens varied between 28.09 and 53.64. Arquero et al. [28] defined the "Marcona" flowers as small and elongated. In our case, the flowers of this last cultivar had a medium size in relation to the rest of cultivars included in the study.

According to Felipe [26], despite there being important differences among cultivars, almond leaves are generally narrow, long and pointed. Leaf characteristics are also affected by environmental conditions and the general health and vigor of the plant [47]. Moreover, leaf size also varies with position, with shoot leaves tending to be large and spur leaves small [48]. Moreover, Grasselly [49] have pointed out that wild cultivars generally have very small leaves, a probable adaptation to the xerophytic conditions under which these cultivars evolved. This was probably the case for the "Bravía" cultivar. Its leaves had the lowest leaf blade length/width ratio (3.76).

Regarding fruit parameters, Socias i Company et al. [46] also observed that the almond parameters are highly variable depending on the cultivar. Moreover, they indicated that traditional cultivars may produce dry fruits of only $2-3 \mathrm{~g}$. This was the case with the cultivars "Bravía" (2.72 g), "Cascafina" ( $3.17 \mathrm{~g}$ ) and "De Convite" (3.86 g). It is important to note that the first cultivar showed small nuts (geometric mean diameter $=18.86 \mathrm{~mm}$, surface area $=11.17 \mathrm{~cm}^{2}$ and volume $=2.42 \mathrm{~cm}^{3}$ ) and the other two had thin and soft shells, respectively. Sorkheh et al. [50] considered small fruit size as one of the most common obstacles to the use of the bitter cultivars for breeding. The weight registered by the almonds of the "Gorda José" cultivar was much higher $(16.35 \mathrm{~g})$. It might be said that the nuts of this traditional cultivar are among the heaviest (usual maximum weight of $20 \mathrm{~g}$ [46]). Other researchers who have also characterized "Marcona" almonds include Melhaoui et al. [24]. Their productive results were slightly lower than those recorded in this work $\left(\right.$ almond weight $=3.58 \mathrm{~g}$, geometric diameter $=21.54 \mathrm{~mm}$, volume $=5.28 \mathrm{~cm}^{3}$ ). These results could have been due to the arid conditions of the cultivation area. With respect to the sphericity, all the nuts were more or less elongated (mean value $=71.24 \%$ ). Aydin [51] also reported mean sphericity values of almond nuts close to $70 \%$. The "Marcona" cultivar showed the roundest nuts (79\%). This result agrees with that obtained by Melhaoui et al. [24] (79.24\%). Muncharaz [52] also identified "Desmayo Largueta" nuts as elongated. Finally, an important number of cultivars showed hard or very hard stony shells. This is due to the fact that hard shells are preferred in the Mediterranean region since the cultivars then seem to be more adapted to non-irrigated conditions, and more resistant to depredation by birds and penetration by insect larvae damaging the kernel. Furthermore, the nuts can be stored for a long time, with reduced problems of becoming rancid or excessively dry, thus allowing their sale throughout the year [46]. Moreover, Aydin [51] also stated that the rupture strength of almond nuts decreased with increasing moisture content. Felipe [26], Mañas et al. [53], Muncharaz [52], Arquero et al. [28] and Batlle et al. [47] also reported that the dry fruits of "Marcona", "Desmayo Largueta" and "Pestañeta" were very hard.

With regard to the kernel, its size and weight are cultivar traits. In general, it can be said that the average weight and size of the almond kernels were quite high (1.36 $\mathrm{g}$ and $0.97 \mathrm{~cm}^{3}$ ). "Marcelina" and "Gorda José" were the cultivars that registered the largest 
$\left(>1.10 \mathrm{~cm}^{3}\right)$ and heaviest ( $\left.>1.94 \mathrm{~g}\right)$ kernels. Socias i Compañy et al. [46] indicated that the range of kernel weight varies between 0.5 to $1.5 \mathrm{~g}$, those that exceed $1.2 \mathrm{~g}$ being preferred for most uses. They also commented that the general trend in the industry is the preference for large kernels in order to facilitate and cheapen the processes of cracking and blanching. Nonetheless, for some special confectioneries very small sizes are chosen, as well as those with definite shapes. For sugared almonds ("peladillas" or dragées) and for chocolate almonds, large kernels are selected, preferably round to reduce the layer of sugar or chocolate covering the kernel. For chocolate bars, small and particularly flat kernels are preferred to ensure that they are covered by the chocolate and that the thickness of the bar is maintained. In addition, there is a strong environmental and seasonal effect on size, including crop load, tree vigor, soil moisture and weather patterns [47]. With respect to kernel yield, the average value was around to $23 \%$. Concretely, "Marcona" almonds had a shelling percentage of $22 \%$. Identical results for nuts of this cultivar were recorded by Melhaoui et al. [24]. Muncharaz [52] also indicated that the shelling percentages for "Marcona" and "Desmayo Largueta" almonds ranged between 22-28\% and 24-28\%, respectively. With regard to the sphericity, the kernel values (average data $=59.10 \%$ ) were lower than those recorded for the nuts. In this sense, Gradziel and Lampinen [54] indicated that very large-sized kernels often bring increased market prices but appear to be associated with lower final tree yields. Felipe [26], Muncharaz [52], Arquero et al. [28] and Melhaoui et al. [24] also defined the "Marcona" kernel as globular and rounded. In addition, a significant number of the almond cultivars showed low percentages of double kernels, among them, the "Marcona" nuts, with a double kernels ratio of $4 \%$. Similar results $(0-5 \%)$ were obtained for the fruits of this cultivar by Melhaoui et al. [24] and Arquero et al. [28]. However, there is a slight difference between the value recorded in this study and that reported by Muncharaz [52] for "Desmayo Largueta" almonds (14\% and 2\%, respectively). In this regard, Batlle et al. [47] have noted that, although double kernels are considered to be a negative trait, lowering crop value, organoleptic quality does not appear to be affected. In this sense, Grasselly and Crossa-Reynaud [55] have pointed out a possible dominance effect with strong seasonal differences. Low temperatures before blooming [56] or at flowering time $[57,58]$ have been mentioned as promoting higher percentages of double kernels. In relation to taste, an important number of cultivars had sweet kernels. According to Batlle et al. [47], the main trend under almond domestication was selection of types with sweet, non-poisonous seeds. Most cultivated almonds produce sweet kernels, but some have a slightly bitter flavor. This was probably the case with the "Recia" cultivar. A mild bitter flavor can be detected in some cultivars and can be considered pleasing in some special confectioneries. It is also important to point out that the sweet or bitter taste depends on the cultivar, so all the fruits of each cultivar will be sweet or bitter, regardless of the genotype of the pollen parent. Finally, regarding all these agromorphological fruit parameters, Socias i Company et al. [59] have pointed out that, although the physical traits of the almond do not affect the sensory characteristics of the almond kernel, they are very important for the processing industry and must be taken into account in the ensemble of requirements for any cultivar, together with the production and consumer sectors.

With respect to chemical characterization of the almonds, there were important differences among cultivars. In general, almond kernels are a rich lipid source, essentially composed of mono- and polyunsaturated fatty acids. Concretely, the average value of lipid fractions for the almonds analyzed was $52.42 \%$. This result is in agreement with that reported by Kodad [60] for Spanish almond cultivars (40-67\% oil content of the kernel dry weight). As previously mentioned, "Peta", "Cascafina" and "Mollar de Arribes" were the cultivars that presented the highest levels of lipids in the kernels ( $>56 \%)$. This lipid content is a very important factor in the confectionery industry because higher oil contents result in less water absorption by the almond paste [61]. Kernels with a high percentage of oil can be used to produce nougat or to extract oil, which is used in the cosmetic and pharmaceutical industries. However, kernels with a low percentage of oil are required to produce almond 
milk [62], almond flour and several kinds of food because of their correlated high protein content [63].

Protein was the second major chemical component of the almond kernels after the lipid fraction. Its average content was $21.13 \%$, with values above $23 \%$ in the cultivars "Bravía", "Molar de Chavés", "Portuguesa", "Picuda" and "Esperanza". This average result is in agreement with that reported by Kodad [60] for Spanish almond cultivars (15.7-21.1\% protein content of the kernel dry weight). According to Alessandroni [61], protein content is inversely correlated with the lipid fraction and the ratio between these two components (R1: \% lipids/\% protein) is important in the preparation of some processed products because the absorption of water by almond paste is dependent on the balance between these two components. For marzipan production, a low index would be desired, whereas for nougat production, a high index would be preferred.

Dietary fiber was the third major chemical component of the almond kernels. Its average content was $15.10 \%$. Ruggeri et al. [64] reported slightly lower dietary fiber contents than those registered in this study for Italian almond cultivars (11-14\% dietary fiber content of the kernel dry weight). These contents have positively effects on colonic health and cholesterol levels [65].

The average content of carbohydrates was 3.89\%. It can be observed that these results for kernel carbohydrates composition again agree with those reported by Kodad [60] for Spanish almond cultivars (1.8-7.6\% carbohydrates content of the kernel fresh weight). In this sense, it is also important to point out that sugars, starch and some sugar alcohols are the only carbohydrate forms present in the almond kernels that can be digested, absorbed and metabolized by humans to provide a source of energy [60]. Moreover, soluble sugars, while present in relatively low amounts, are sufficient to make kernels sweet-tasting [66].

In addition, the average content of ash was $3.60 \%$. The almond kernel is considered a good source of mineral elements, playing an important role in human health [60]. Regarding this, Romojaro et al. [67] and Saura-Calixto et al. [65] also reported low variability for this parameter in Spanish almond cultivars (3.05-3.45\%).

In relation to energy, the high nutritive value of almond kernels arises mainly from their high lipid content, which constitutes an important source of calories [68]. The average caloric content of the kernels analyzed was $571.70 \mathrm{kcal}$ per $100 \mathrm{~g}$ fresh weight of edible portion. A similar result (578 kcal/100 g FW) was reported by Gradziel [69].

Finally, the growth habits were highly variable among cultivars, ranging from very upright or upright (the "Agapitina" cultivar) to completely drooping (the "Desmayo Largueta" cultivar, among others). Socias i Company et al. [46], Mañas et al. [53], Felipe [26] and Muncharaz [52] have also observed that "Desmayo Largueta" has a fairly common drooping growth habit. Arquero et al. [28] classified the vegetative habits of "Marcona" and "Desmayo Largueta" cultivars as spreading. In this regard, Espada and Connell [70] have indicated that upright trees have better shaking efficiency than trees with roundish or hanging canopies. However, when the habit is very erect, the tree may have a canopy with insufficient spread. This makes orchard management difficult but may be useful in new developments of high density plantings. Generally an upright to spreading habit is preferable, as it facilitates formation of the tree and mechanization of the different operations [46]. Thus, the tree habit of modern European cultivars is generally spreading [47].

\subsection{Statistical Analyses}

Principal component analysis (PCA) results showed that more than $54 \%$ of the variability observed was explained by the first three components. These results agree with those obtained by Gouta et al. [71], Čolić et al. [72] and Khadivi-Khub and Etemadi-Khah [73] for almond cultivars of the Mediterranean area. PCA revealed that the first three components explained comparable values (from $34 \%$ to $57 \%$ ) of the total variation, based on morphological and biochemical traits. Furthermore, Lansari et al. [74], Talhouk et al. [11], and Sorkheh et al. [50], who used a similar analysis to compare kernel, nut and leaf characters 
in different almond collections, found that the variables contributing to nut and kernel size were more important than leaf traits.

By analyzing the dendrogram, and taking into account all the results shown above, a series of synonymies among the almond cultivars can also be detected. Such is the case with "Pestaneta de Bragança" and "Pestañeta de Arribes", and with "Verdeal" and "Verdinal". Significant similarities were observed between the cultivars for these two cases of synonymies for all agromorphological and chemical traits. By contrast, a homonym was also detected: "Molar de Chavés" and "Mollar de Arribes". Despite their major agromorphological and chemical differences, both names are often used interchangeably by some growers.

The results of the PCA and cluster analysis showed that agromorphological and chemical analysis can provide reliable information on the variability in almond genotypes. In correspondence with our findings, Ledbetter and Shonnard [75], Talhouk et al. [11], Sorkheh et al. [76], Zeinalabedini et al. [77] and Khadivi-Khub and Etemadi-Khah [73] have also shown that morphological evaluation is an efficient tool for characterization of almond germplasm and for species distinction. The overall analysis of all traits illustrates a wide diversity that may have important implications for management of genetic resources.

\section{Conclusions}

Twenty-four traditional almond cultivars grown in the central-western Iberian Peninsula, all of them clearly in decline or close to extinction, were characterized from the agromorphological and chemical points of view. Some of the cultivars showed distinctive and interesting agronomical characteristics from a commercial point of view, such as high yields and high quality fruit. This was the case for the almond cultivars called "Gorda José" and "Marcelina". Their fruits were quite heavy (nuts: $>9.1 \mathrm{~g}$; kernels: $>1.9 \mathrm{~g}$ ), with very low percentages of double kernels $(<3 \%)$ and high nutritional value $(>50 \%$ lipids; $>21 \%$ proteins). The results of PCA and cluster analysis showed that agromorphological and chemical analysis can provide reliable information on the variability in almond genotypes. Two synonymies ("Pestaneta de Bragança" and "Pestañeta de Arribes"; "Verdeal" and "Verdinal") and one homonym ("Molar de Chavés" and "Mollar de Arribes") were also detected. This work constitutes an important step in the conservation of genetic almond resources in the central-western Iberian Peninsula.

Author Contributions: Conceptualization, R.P.-S. and M.R.M.-C.; Methodology, R.P.-S. and M.R.M.C.; Software, R.P.-S.; Validation, R.P.-S. and M.R.M.-C.; Investigation, R.P.-S. and M.R.M.-C.; Data curation, R.P.-S.; Writing—original draft preparation, R.P.-S.; Writing—review and editing, R.P.-S. and M.R.M.-C.; Project administration, R.P.-S.; Funding acquisition, R.P.-S. All authors have read and agreed to the published version of the manuscript.

Funding: This research was supported by the Biodiversity Foundation of the Spanish Ministry of Environment and Rural and Marine Affairs (MARM) and the Spanish Federation of Municipalities and Provinces (FEMP). (Project reference: MARM-FB-2008-012).

Institutional Review Board Statement: Not applicable.

Acknowledgments: The authors thank the almond growers of the "Arribes del Duero" (Salamanca, Spain) and "Trás-os-Montes e Alto Douro" (Bragança, Portugal) for their major contributions to this work.

Conflicts of Interest: The authors declare no conflict of interest.

\section{References}

1. Watkins, R. Cherry, plum, peach, apricot and almond. Prunus spp. In Evolution of Crop Plants; Simmonds, N.W., Ed.; Cambridge University Press: London, UK, 1976; pp. 242-247.

2. Mohamed, B.S. The contribution of Prunus webbii to almond evolution. PGR Newsl. 2004, 140, 9-13.

3. Kester, D.E.; Gradziel, T.M. Almonds. In Fruit Breeding-Volume III: Nuts; Janick, J., Moore, J.N., Eds.; Wiley-Blackwell: New York, NY, USA, 1996; pp. 1-96.

4. Duke, J.A. Handbook of Nuts; CRC Press: Boca Ratón, FL, USA, 2000. 
5. Food and Agriculture Organization (FAO). Faostat: Agriculture Data. 2019. Available online: http://www.fao.org/faostat/es/ \#data/QC (accessed on 28 April 2021).

6. Gradziel, T.M.; Curtis, R.; Socias i Company, R. Production and growing regions. In Almonds. Botany, Production and Uses; Socias i Company, R., Gradziel, T.M., Eds.; CABI: Wallingford, UK, 2017; pp. 70-86.

7. Vargas, F.J. El Almendro en la Provincia de Tarragona; Excma. Diputación Provincial de Tarragona, Servicio Agropecuario Provincial: Tarragona, Spain, 1975.

8. García, J.E.; Egea, L.; Egea, J.; Berenguer, T. Programme d'amélioration de l'amandier au C.E.B.A.S. de Murcie. Options Méditerranéennes 1985, 85, 7-8.

9. García, J.E.; Egea, L.; Egea, J.; Berenguer, T. Étude et sélection de variétés d'amandier dans le sudest de l’Espagne. Rapp. EUR 1988, 11557, 33-46.

10. Nieddu, G.; Chessa, I.; Pala, M.; Lovicu, G. Evaluation of almond germplasm in Sardinia: Further observations. Acta Hortic. 1994, 373, 135-140. [CrossRef]

11. Talhouk, S.N.; Lubani, R.T.; Baalbaki, R.; Zurayk, R.; Al Khatib, A.; Parmaksizian, L.; Jaradat, A.A. Phenotypic diversity and morphological characterization of Amygdalus L. species in Lebanon. Genet. Resour. Crop Evol. 2000, 47, 93-104. [CrossRef]

12. De Giorgio, D.; Polignano, G.B. Evaluation the biodiversity of almond cultivars from a germplasm collection field in Southern Italy. In Sustaining the Global Farm; Stott, D.E., Mohtar, R.H., Steinhardt, G.C., Eds.; ISCO: West Lafayette, IN, USA, 2001; pp. 305-311.

13. Kaska, N.; Kafkas, S.; Padulosi, S.; Wassimi, N.; Ak, B.E. Characterization of nuts species of Afghanistan: I-Almond. Acta Hortic. 2006, 726, 147-156. [CrossRef]

14. Chalak, L.; Chehade, A.; Kadri, A. Morphological characterization of cultivated almonds in Lebanon. Fruits 2007, 62, 177-186. [CrossRef]

15. Arslan, S.; Vursavuş, K.K. Physico-mechanical properties of almond nut and its kernel as a function of variety and moisture content. Philipp. Agric. Sci. 2008, 91, 171-179.

16. Jahanban, A.; Jahanban, R.; Jamei, R.; Jahanban, A. Morphology and physicochemical properties of 40 genotypes of almond (Amygdalus communis L.) fruits. Eur. J. Exp. Biol. 2012, 2, 2456-2464.

17. Hanine, H.; Zinelabidine, L.H.; Hssaini, H.; Nablousi, A.; Ennahli, S.; Latrache, H.; Zahir, H. Pomological and biochemical characterization of almond cultivars in Morocco. Turk. J. Agric. Nat. Sci. 2014, 1, 743-753.

18. Hanine, H.; Zinelabidine, L.H.; H'ssaini, H.; Ennahli, S.; Latrache, H.; Hmid, I. Phenotypic, morphological diversity and biochemical characterization of 14 almond cultivars from Morocco. Moroc. J. Chem. 2015, 3, 394-406.

19. Ardjmand, A.; Piri, S.; Imani, A.; Piri, S. Evaluation of morphological and pomological diversity of 62 almond cultivars and superior genotypes in Iran. J. Nuts 2014, 5, 39-50.

20. Kodad, O.; Estopañán, G.; Fagroud, M.; Juan, T.; Socias i Company, R. Physical and chemical traits of almond kernels of the local almond populations in Morocco: Commercial and industrial end-uses. Acta Hortic. 2014, 1028, 233-238. [CrossRef]

21. Kodad, O.; Lebrigui, L.; El-Amrani, L.; Socias i Company, R. Physical fruit traits in Moroccan almond seedlings: Quality aspects and post-harvest uses. Int. J. Fruit Sci. 2015, 15, 36-53. [CrossRef]

22. Sepahvand, E.; Khadivi-Khub, A.; Momenpour, A.; Fallahi, E. Evaluation of an almond collection using morphological variables to choose superior trees. Fruits 2015, 70, 53-59. [CrossRef]

23. Giordani, E.; Berti, M.; Rauf Yaqubi, M. Phenotypic characterisation of almond accessions collected in Afganistan. Adv. Hortic. Sci. 2016, 30, 207-216.

24. Melhaoui, R.; Addi, M.; Houmy, N.; Abid, M.; Mihamou, A.; Serghini-Caid, H.; Sindic, M.; Elamrani, A. Pomological characterization of main almond cultivars from the North Eastern Morocco. Int. J. Fruit Sci. 2019, 19, 413-422. [CrossRef]

25. Montero-Riquelme, F.J. Caracterización Morfológica del Almendro; Servicio de Publicaciones de la Universidad de Castilla-La Mancha: Murcia, Spain, 1993.

26. Felipe, A.J. El Almendro. Volume I. El Material Vegetal; Mira Editores: Zaragoza, Spain, 2000.

27. Cordeiro, V.; Monteiro, A.; Oliveira, M.; Ventura, J. Study of some physical characters and nutritive composition of the Portuguese's (local) almond varieties. Options Méditerranéennes 2001, 56, 333-337.

28. Arquero, O.; Lovera, M.; Navarro, A.; Viñas, M.; Salguero, A.; Serrano, N. Hábitos de Vegetación y Respuesta a la Intensidad de la Poda de Formación de las Principales Variedades de Almendro; Consejería de Agricultura y Pesca, Junta de Andalucía: Sevilla, Spain, 2008.

29. Kodad, O.; Socias i Company, R. Variability of oil content and of major fatty acid composition in almond (Prunus amygdalus Batsch) and its relationship with kernel quality. J. Agric. Food Chem. 2008, 56, 4096-4101. [CrossRef] [PubMed]

30. Kodad, O.; Socias i Company, R. Fruit quality in almond as related to the type of pollination in self-compatible genotypes. J. Am. Soc. Hortic. Sci. 2008, 133, 320-326. [CrossRef]

31. Vargas, F.J.; Romero, M.; Clavé, J.; Alegre, S.; Miarnau, X. Variedades de Almendro IRTA; Ibercaja: Madrid, Spain, 2009.

32. Kodad, O.; Mamouni, A.; Lahlo, M.; Socias i Company, R. Implicación commercial e industrial de la variabilidad del contenido en aceite y proteína y de los caracteres físicos del fruto y de la pepita del almendro en las condiciones climáticas mediterráneas. Inf. Técnica Económica Agrar. 2011, 107, 300-314.

33. Kodad, O.; Estopañán, G.; Juan, T.; Socias i Company, R. Genetic diversity in an almond germplasm collection: Application of a chemometric approach. Acta Hortic. 2013, 976, 237-242. [CrossRef] 
34. Kodad, O.; Estopañán, G.; Juan, T.; Alonso, J.M.; Espiau, M.T.; Socias i Company, R. Oil content, fatty acid composition and tocopherol concentration in the Spanish almond genebank collection. Sci. Hortic. 2014, 177, 99-107. [CrossRef]

35. Ramos, M.A.; Costa, M.G. Characteristics of the Fruits of Some Varieties of Almond Grown in the Algarve Region; Faculty of Sciences and Technology, University of Algarve: Faro, Portugal, 2014.

36. UPOV. Guidelines for the Conduct of Test for Distinctness, Homogeneity and Stability. Almond (Prunus amygdalus Batsch); International Union for the Protection of New Varieties of Plants: Geneva, Switzerland, 1978.

37. IPGRI. Descriptors List for Almond (Prunus amygdalus) (Revised); International Board for Plant Genetic Resources: Rome, Italy, 1985.

38. Mohsenin, N.N. Physical Properties of Plant and Animal Materials; Gordon and Breach Science Publishers: New York, NY, USA, 1978.

39. Baryeh, E.A. Physical properties of Bambara groundnuts. J. Food Eng. 2001, 47, 321-326. [CrossRef]

40. Güner, M.; Dursun, E.; Dursun, I.G. Mechanical behavior of the hazelnut under compression loading. Biosyst. Eng. 2003, 4, 485-491. [CrossRef]

41. Olajide, J.O.; Igbeka, J.C. Some physical properties of groundnut kernels. J. Food Eng. 2003, 58, 201-204. [CrossRef]

42. Jain, R.K.; Bal, S. Physical properties of pearl millet. J. Agric. Eng. Res. 1997, 66, 85-91. [CrossRef]

43. Özgüven, F.; Vursavuş, K. Some physical, mechanical and aerodynamic properties of pine (Pinus pinea) nuts. J. Food Eng. 2005, 68, 191-196. [CrossRef]

44. AOAC. Official Methods of Analysis, 16th ed.; Association of Official Analytical Chemists: Arlington, TX, USA, 1995.

45. Iezzoni, A.F.; Pritts, M.P. Applications of principal components analysis to horticultural research. HortScience 1991, 26, 334-338. [CrossRef]

46. Socias i Company, R.; Ansón, J.M.; Espiau, M.T. Taxonomy, botany and physiology. In Almonds. Botany, Production and Uses; Socias i Company, R., Gradziel, T.M., Eds.; CABI: Wallingford, UK, 2017; pp. 1-42.

47. Batlle, I.; Dicenta, F.; Socias i Company, R.; Gradziel, T.M.; Wirthensohn, M.; Duval, H.; Vargas, F.J. Classical genetics and breeding In Almonds. Botany, Production and Uses; Socias i Company, R., Gradziel, T.M., Eds.; CABI: Wallingford, UK, 2017 ; pp. 111-148.

48. Gradziel, T.M. Almond (Prunus dulcis). In Breeding of Plantation Crops; Priyadarshan, M., Jain, S.M., Eds.; Springer: Berlin, Germany, 2008; pp. 1-33.

49. Grasselly, C. Les espèces sauvages d'amandiers. Options Méditerranéennes 1976, 32, $28-43$.

50. Sorkheh, K.; Shiran, B.; Rouhi, V.; Asadi, E.; Jahanbazi, H.; Moradi, H.; Gradziel, T.M.; Martínez, P. Phenotypic diversity within native Iranian almond (Prunus spp.) species and their breeding potential. Genet. Resour. Crop Evol. 2009, 56, 947-961. [CrossRef]

51. Aydin, C. Physical properties of almond nut and kernel. J. Food Eng. 2003, 60, 315-320. [CrossRef]

52. Muncharaz, M. El Almendro-Manual Técnico; Mundi-Prensa: Madrid, España, 2004.

53. Mañas, F.; López, P.; Rodríguez, A. Mejora genética de material autóctono de almendro en Castilla-La Mancha para floración tardía y calidad. Mem. ITAP 2000, 2000, 293-325.

54. Gradziel, T.; Lampinen, B. Defining the limits of almond productivity to facilitate marker assisted selection and orchard management. Acta Hortic. 2011, 912, 47-52. [CrossRef]

55. Grasselly, C.; Crossa-Raynaud, P. L'Amandier; G.P. Maisonneuve et Larose: Paris, France, 1980.

56. Egea, J.; Burgos, L. Double kernel fruits in almond (Prunus dulcis Mill.) as related to pre-blossom temperatures. Ann. Appl. Biol. 1994, 126, 163-168. [CrossRef]

57. Rikhter, A.A. Ways and methods of almond breeding (in Russian). Tr. Gos. Nikitsk. Bot. Sada 1969, 43, 81-94.

58. Spiegel-Roy, P.; Kochba, J. The inheritance of bitter and double kernel characters in the almond (Prunus amydgalus Batsch). Z. Pflanz. 1974, 71, 319-329.

59. Socias i Company, R.; Alonso, J.M.; Kodad, O. Fruit quality in almond: Physical aspects for breeding strategies. Acta Hortic. 2009, 814, 475-480. [CrossRef]

60. Kodad, O. Chemical composition of almond nuts. In Almonds. Botany, Production and Uses; Socias i Company, R., Gradziel, T.M., Eds.; CABI: Wallingford, UK, 2017; pp. 428-448.

61. Alessandroni, A. Le mandorle. Panifazione Pastic. 1980, 8, 67-71.

62. Ramusino, F.C.; Intonti, R.; Stachini, A. Analisi del latte di mandorle e dello sciroppo di orzata. Bolletino Dei Lab. Chem. Prov. 1961, $12,491-504$.

63. Longhi, S. High-Protein Animal Feed. Ital. Pat. 1952, 470, 433.

64. Ruggeri, S.; Cappelloni, M.; Gambelli, L.; Nicoli, S.; Carnovale, E. Chemical composition and nutritive value of nuts grown in Italy. J. Food Sci. 1998, 3, 243-251.

65. Saura-Calixto, F.; Bauzá, M.; Martínez, F.; Argamentería, A. Amino acids, sugars, and inorganic elements in the sweet almond. J. Agric. Food Chem. 1981, 29, 509-511. [CrossRef] [PubMed]

66. Schirra, M. Postharvest technology and utilization of almonds. Hortic. Rev. 1997, 20, 267-292.

67. Romojaro, F.; García, J.E.; López-Andreu, F.J. Estudio sobre la composición química de variedades de almendra del sureste español. An. Edafol. Agrobiol. 1977, 36, 121-131.

68. Sabate, J.; Hook, D.G. Almonds, walnuts and serum lipids. In Lipids in Human Nutrition; Spiller, G.A., Ed.; CRC Press: Boca Raton, FL, USA, 1996; pp. 137-144.

69. Gradziel, T.M. History of cultivation. In Almonds. Botany, Production and Uses; Socias i Company, R., Gradziel, T.M., Eds.; CABI: Wallingford, UK, 2017; pp. 43-69. 
70. Espada, J.L.; Connell, J.H. Almond harvesting. In Almonds. Botany, Production and Uses; Socias i Company, R., Gradziel, T.M., Eds.; CABI: Wallingford, UK, 2017; pp. 406-427.

71. Gouta, H.; Mars, M.; Gouiaa, M.; Ghrab, M.; Zarrouk, M.; Mliki, A. Genetic diversity of almond (Prunus amygdalus Batsch) in Tunisia: A morphological traits analysis. Acta Hortic. 2011, 912, 351-358. [CrossRef]

72. Čolić, S.; Rakonjac, V.; Zec, G.; Nicolić, D.; Fotirić, M. Morphological and biochemical evaluation of selected almond [Prunus dulcis (Mill.) D.A.Webb] genotypes in northern Serbia. Turk. J. Agric. For. 2012, 36, 429-438.

73. Khadivi-Khub, A.; Etemadi-Kah, A. Phenotypic diversity and relationships between morphological traits in selected almond (Prunus amygdalus) germplasm. Agrofor. Syst. 2015, 89, 205-216. [CrossRef]

74. Lansari, A.; Iezzoni, A.F.; Kester, D.E. Morphological variation within collection of Moroccan almond clones and Mediterranean and North American cultivars. Euphytica 1994, 78, 27-41.

75. Ledbetter, C.A.; Shonnard, C.B. Evaluation of selected almond (Prunus dulcis (Miller) D.A. Webb) germplasm for several shell and kernel characteristics. Fruit Var. J. 1992, 46, 79-82.

76. Sorkheh, K.; Shiran, B.; Khodambashi, M.; Moradi, H.; Gradziel, T.M.; Martinez-Gómez, P. Correlations between quantitative tree and fruit almond traits and their implications for breeding. Sci. Hortic. 2010, 125, 323-331. [CrossRef]

77. Zeinalabedini, M.; Sohrabi, S.; Nikoumanesh, K.; Imani, A.; Mardi, M. Phenotypic and molecular variability and genetic structure of Iranian almond cultivars. Plant Syst. Evol. 2012, 298, 1917-1929. [CrossRef] 